\title{
A Pressure-based Compressible-Liquid Flow Model for Computation of Instantaneous Valve Closure in Pipes
}

\author{
R. Jishnu Chandran ${ }^{1 *}$ and A. Salih ${ }^{2}$ \\ ${ }^{1,2}$ Department of Aerospace Engineering, \\ Indian Institute of Space Science and Technology, \\ Thiruvananthapuram-695547, Kerala, India \\ E-mail: *jishnuchnadranr@gmail.com
}

\begin{abstract}
A generalized, pressure-based compressible-liquid flow model is proposed for the isothermal low-speed flow of liquids. The flow model incorporates dedicated equations of state for liquids into the pressure-based solvers to simulate the compressible effects in liquids. The model's capability to handle compressible liquid flow problems is evaluated against the well-established density-based water-hammer model. The isothermal flow problem of an instantaneous valve closure in an irrigation pipe and the associated flow transients are numerically solved using the proposed model by employing the Tait equation of state in conjunction with a segregated pressure-based solver algorithm. The transient flow problem is solved for a range of operating pressures, and the surge in pressure and variation of other flow properties and their interrelations are studied in detail. The proposed model could successfully capture the entire physics of the problem, including the compressible modeling of the liquid involved and could produce high accuracy numerical results. The results suggest that the pressure-based compressible-liquid flow model is a reliable and computationally inexpensive numerical tool for isothermal low-speed compressible liquid flow computations.
\end{abstract}

Keywords: Instantaneous Valve Closure; Pressure-based algorithm; Compressible Liquid; Tait Equation of State; Pressure Surge, Pipe Flow

\section{INTRODUCTION}

Isothermal liquid flows are mostly modeled as incompressible even for the pressure ranges well above the ambient conditions, where we treat the liquid density as a constant. The Mach numbers associated with such cases remain very small, and hence the compressible solvers (density-based) are not employed for their modeling. The incompressible pressure-based solvers are widely employed in modeling such low-speed flow situations. The pressure-based solvers are computationally less expensive than the density-based solvers due to the non-enforcement of the acoustic time scale, and they treat the fluid density to be constant.

There are certain flow situations where the flow physics encounters pressure magnitudes much higher than the ambient pressure where one can expect variations in the liquid's density with local pressure. The conventional pressure-based methods, if employed to model such flow physics, cannot capture the variations in density. In the present work, a simple and generalized 'pressure-based compressible-liquid flow model' is proposed for modeling the compressibility effects in a liquid using a pressurebased solver, especially for isothermal cases where the fluid density varies only with pressure. Various equations of state for liquids are available in literature for relating the liquid density to its temperature and pressure.

The physical problem considered in this study is the instantaneous valve closure in a large diameter irrigation pipe, which is a case of a low-speed isothermal flow of a liquid encountering substantial pressure variations. The proposed 'pressure-based compressible-liquid flow model' employing a dedicated equation of state for water along with a segregated pressure-based solver of a commercial 
software package, is used to simulate this transient flow problem, involving compressible effects in liquids. The performance and accuracy of this model is evaluated by comparing the results with those obtained using a wellestablished density-based method for handling such class of flow problems, viz., the one-dimensional water hammer equations (Students 1903, Guinot 2003).

\section{THE PRESSURE-BASED COMPRESSIBLE- LIQUID FLOW MODEL}

Pressure-based methods are primarily developed for lowspeed incompressible flows. Over the years, numerous advancements in the formulation of this method enabled its application over a broader range of flow-speeds and conditions. A comprehensive review of various pressurebased methods is available in (Acharya et al. 2007). Advanced pressure-based methods are reported in the works (Chen 1989, Rincon and Elder 1997, Moukalled and Darwish 2001, Shterev and Stefanov 2010, Chen and Przekwas 2010, Lourier et al. 2012, Miller et al. 2013, Kambrath and Cary 2014, Miettinen and Siikonen 2015, Xiao et al. 2017, Kraposhin et al. 2018, Denner 2018), most of which are capable of modeling a wide range of compressible flows.

The present work proposes a generalized 'pressure-based compressible-liquid flow model.' The model can handle the compressible effects in liquid under isothermal highpressure applications. The proposed model achieves this by using dedicated equations of state (EOS) for liquids in conjunction with any suitable pressure-based algorithms. This model thus utilizes the computational efficiency of the pressure-based algorithms to simulate compressible liquid flow cases. This study is limited to isothermal flow cases, and hence an isothermal EOS is preferred, which directly relates the fluid density to its pressure. Even a non-isothermal EOS could be used by assigning the temperature a constant value throughout the simulation process. The methodology used by the pressure-based compressible-liquid flow model is described in detail below.

The first step of any typical segregated pressure-based algorithm for the simulation of transient flows is the initialization of flow variables such as pressure and velocity. The proposed model could be used for the computation of compressible liquid flows by incorporating a suitable liquid equation of state into any generic pressure-based algorithm. The initial value of the density of the liquid is estimated using the specific EOS chosen. A segregated solver algorithm for a system of coupled non-linear equations solves the system sequentially in a loop until convergence criteria are met. The flow chart for the pressure-based compressibleliquid flow model using a typical segregated pressure-based algorithm is shown in Fig. 1.

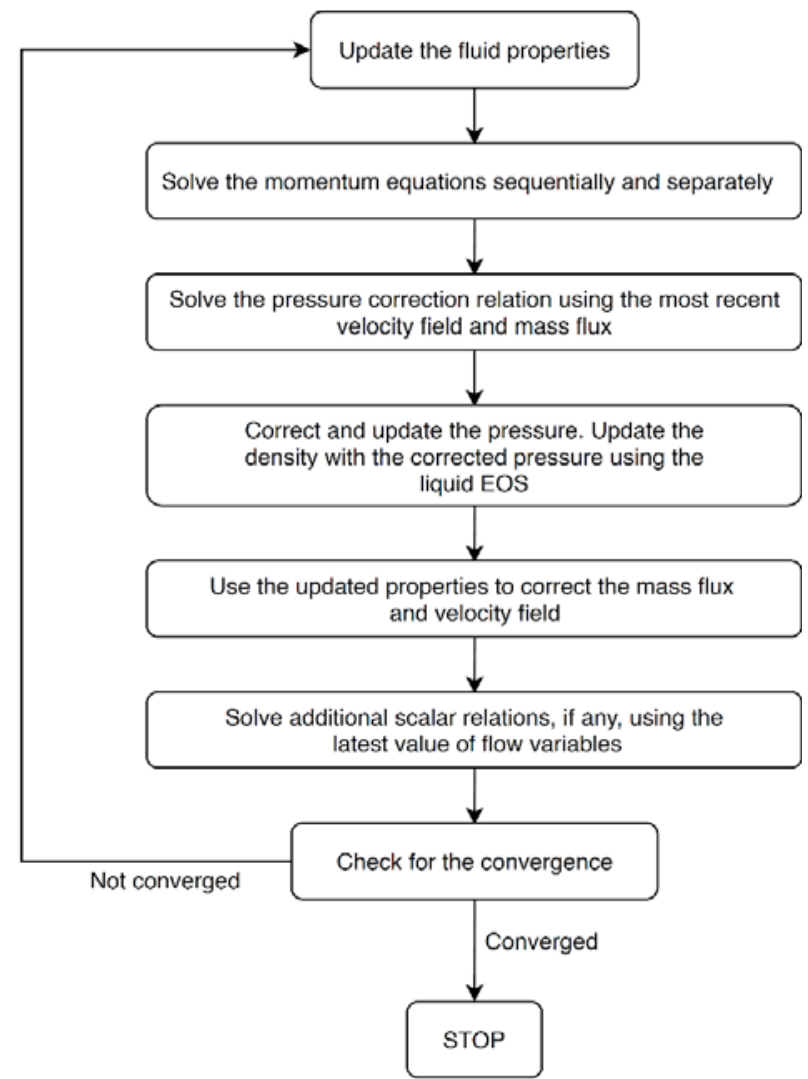

Fig. 1: Flow Chart of the Pressure-based CompressibleLiquid Flow Model using a Typical Segregated PressureBased Algorithm

With the updated values of flow properties, the momentum equations are solved one after the other sequentially to obtain the velocity field and mass flux. The computed values applied to the pressure correction equation provides the correction for pressure. Using this, the pressure is corrected and updated. The updated pressure field is now fed into the equation of state of the liquid to generate the updated density field. Subsequently, the corrected velocity field and mass fluxes are estimated from the updated flow variables. Any scalar quantity related to the flow field, which is yet to be computed, could be estimated using the corresponding relations with the updated flow variables. The solution eventually is checked for convergence. On reaching convergence, the solver loop stops for a steadystate problem, else the loop repeats. For transient flows, the converged values of flow variables are assigned as the initial 
value for the next time step, and then the time marching process continues. It may be noted that whenever there is a correction in pressure, the density is immediately updated using the EOS.

\section{TEST CASE PROBLEM-THE INSTANTANE- OUS VALVE CLOSURE}

The test case chosen for performance analysis of the proposed pressure-based compressible-liquid model is the instantaneous closure of a valve in a constant diameter circular pipe. Instantaneous valve closures are among the worst-case flow transients as they generate maximum surge intensity in pressure in a short duration. The variations observed in the flow variables for such cases are more or less unidirectional as stated in (Ghidaoui et al. 2005), " rapid flow disturbances, planned or accidental, induce spatial and temporal changes in the velocity and pressure fields in pipe systems and such transient flows are essentially unidirectional since the axial fluxes of mass, momentum, and energy are far greater than their radial counterparts". A study on laminar water-hammer (Mitra and Rouleau 2005) and turbulent water-hammer (Vardy and Hwang 2005) also support the unidirectional approach in the problem formulation. Detailed literature on valve induced transients are available in the books (Wylie 2003, Tijsseling and Bergant 2007) and related works are also reported in (Bazargan-Lari et al. 2013, Choi et al. 2015, Kodura 2016).

As a practically relevant problem, an irrigation pipe is chosen for the present study. For the pipe dimension and the permissible flow velocities associated with irrigation pipes, the report on the Pipe Distribution System for Irrigation (Indian National Committee on Irrigation and Drainage 1998) constituted by the Ministry of Water Resources, Govt. of India, is referred. The schematic of the physical domain chosen is shown in Fig. 2.

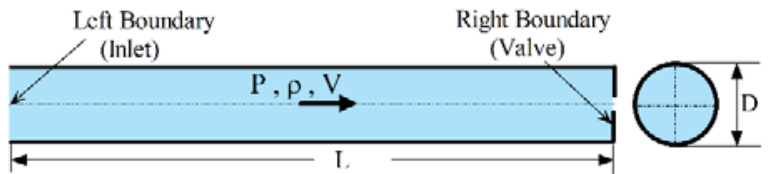

Fig. 2: Schematic of the Physical Domain

The report (Indian National Committee on Irrigation and Drainage 1998) suggests a maximum allowable velocity close to $1.5 \mathrm{~m} / \mathrm{s}$ and the diameter of pipes ranging from $0.3 \mathrm{~m}$ to $0.9 \mathrm{~m}$. A pipe diameter of $\mathrm{D}=0.9 \mathrm{~m}$ and flow velocity $\mathrm{V}=1.5$ $\mathrm{m} / \mathrm{s}$ are selected for this study. An $\mathrm{L}=3.0 \mathrm{~m}$ long pipe with its right end closed is chosen as the domain. Velocity in the flow domain is initialized with a uniform value of $1.5 \mathrm{~m} / \mathrm{s}$. This uniform flow and the closed-end combination simulates the instantaneous closure of the valve. The analysis is carried out for three different operating pressure ranges within the maximum range allowable as per the report viz., 1 bar, 2.5 bar, and 25 bar guage. Solution profiles of the flow variables such as the pressure, fluid density, and flow velocity after 1 millisecond from the instantaneous valve closure are chosen for the comparative study.

\section{NUMERICAL MODEL AND EVALUATION STRATEGY}

\section{NUMERICAL MODEL}

The pressure-based compressible-liquid flow model using the segregated-pressure-based algorithm explained in Section 2, is used to numerically simulate the transient test problem. The viscous terms are excluded from the computational model as the flow Reynolds number is well above 10,00,000 for the configuration considered. A rectangular two-dimensional axisymmetric computational domain of length $3.0 \mathrm{~m}$ and width $0.45 \mathrm{~m}$ is used, as shown in Fig. 3.

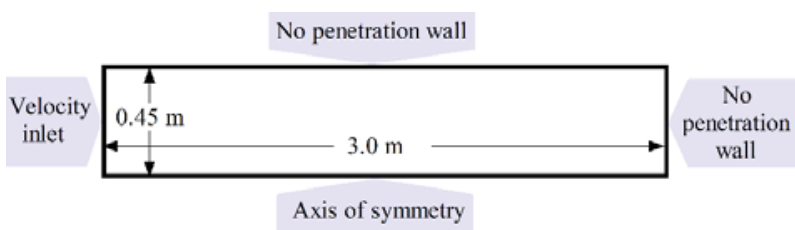

Fig. 3: 2D Axisymmetric Computational Domain and Boundary Types

The boundary treatments are also shown in the figure, where the bottom boundary is the axis of symmetry, and the top face is a wall with no penetration boundary condition. A uniform velocity profile of $1.5 \mathrm{~m} / \mathrm{s}$ is prescribed at the inlet (left) boundary. On the right boundary, a no penetration boundary condition is imposed. This is tantamount to an instantaneous full closure of the valve at the beginning of the computation if the fluid velocity inside the domain is initialized to a uniform value of $1.5 \mathrm{~m} / \mathrm{s}$. The domain is discretized by taking 800 uniform control volumes lengthwise and 50 control volumes in the radial direction. Grid independent results are obtained for all the pressure ranges considered in the study.

Liquid water is modeled to be compressible through the use of the Tait EOS. The Tait EOS for compressible liquids was first introduced by the Scottish mathematician P. G. 
Tait in (Tait 1888), and its historical evolution is presented in (Dymond and Malhotra 1988). This state equation establishes a nonlinear relationship between density and pressure under isothermal conditions for water as in Eq.1 (Saurel et al. 1999).

$$
P=\frac{K_{0}}{\theta}\left[\left(\frac{\rho}{\rho_{O}}\right)^{\theta}-1\right]+P_{0}
$$

Here $P_{0}$ and $\rho_{0}$ are respectively the reference values of liquid pressure and density. Parameter $K_{0}$ is the reference bulk modulus of water, which is taken as $2.2 \times 10^{9} \mathrm{~Pa}$. The density exponent, $\theta$ for water is 7.15. The Tait EOS is an excellent choice for relating the fluid density to pressure as it very accurately predicts the density for a temperature around $300 \mathrm{~K}$. At this temperature range, the density estimates show an average error below $0.25 \%$ and maximum error below $0.70 \%$ over the wide pressure range extending from 1 bar to 10,000 bar (Chandran and Salih 2019).

The momentum fluxes are computed with a second-order accurate upwind scheme. A first-order implicit transient formulation is used to model the time-dependent flow problem. Property gradients are evaluated by the leastsquares cell-based method. The transient simulation is run for a duration of 1 millisecond with a uniform time step size $\Delta t=5 \times 10^{-7} \mathrm{~s}$.

\section{EVALUATION STRATEGY}

The capability of the proposed model for handling the compressibility effects of the liquid, and its performance while simulating a transient flow problem involving a moving compression front, needs a thorough investigation. A well-established density-based method to handle such class of flow problems involving the compressibility effect in liquids is the classical water-hammer formulation. The equations of the water hammer formulation are derived from the conservation of mass and momentum principles. In their correct mathematical form, they are first published in (Joukowsky 1898); however, the theory of water hammer phenomena is generally associated with the name of Allievi (Students 1903). (Guinot 2003) gives a detailed description of the hyperbolic conservation equations for the onedimensional water hammer. The one-dimensional density- based approach detailed by (Guinot 2003) is adopted for solving the instantaneous valve closure problem. This result is chosen as the benchmark for evaluating the performance of the proposed pressure-based compressible-liquid flow model. For the one-dimensional water hammer formulation, the initial fluid density is computed from the standard NIST isothermal database for the specified operating pressure conditions. In this formulation speed of sound is updated with variable fluid density. The one-dimensional computational domain is discretized uniformly into 800 control volumes in the axial direction.

\section{RESULTS AND DISCUSSION}

The flow transients associated with the instantaneous valve closure in a constant diameter pipe is numerically solved for three different operating pressure conditions viz., 1 bar, 2.5 bar, and 25 bar (gauge). The pressure-based compressibleliquid flow model is applied to a 2D axisymmetric domain for simulating the transient flow. This pressure-based compressible flow model employs the Tait EOS for the compressible liquid and a segregated pressure-based algorithm for solving the governing equations of the flow.

For the purpose of illustration, we have used the pressurebased algorithm available in the commercial ANSYS Fluent package. The same flow problem with three different pressures is also solved on a one-dimensional domain using classical water hammer formulation (Guinot 2003). The result obtained using the proposed compressible-liquid model has been compared with that of the density-based classical water-hammer formulation. Figures 4(a) and 4(b) display the pressure and density profiles along the flow axis at a time of one millisecond after the valve's closure.

The property profiles computed using the compressibleliquid model are plotted using solid symbols, and continuous lines show those computed with the classical water-hammer method. It can be seen that the results of the proposed model are in excellent agreement with the results of the benchmark solution of the water hammer problem. It can also be observed that this agreement with the benchmark results is consistent over all the three operating pressure ranges considered. 


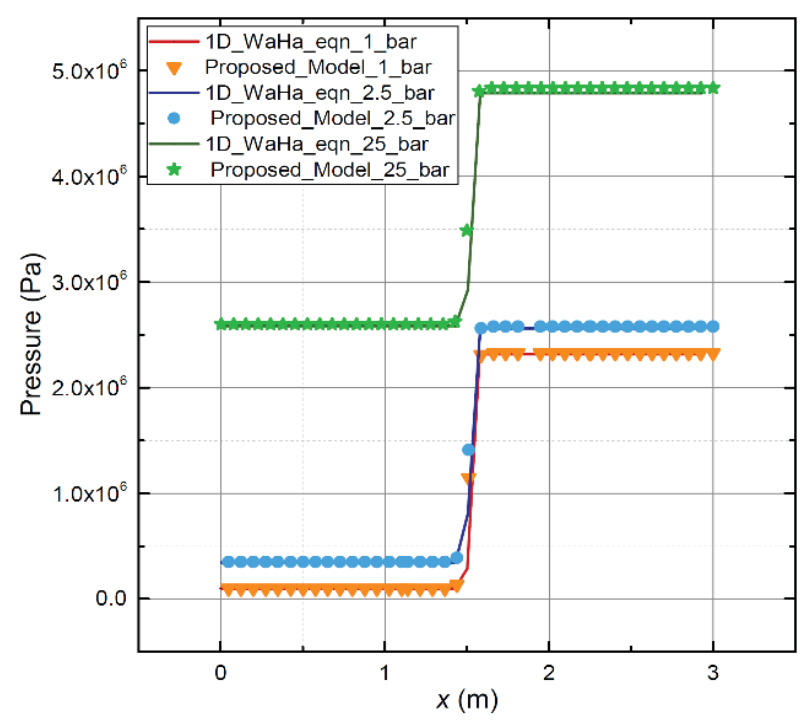

(a)

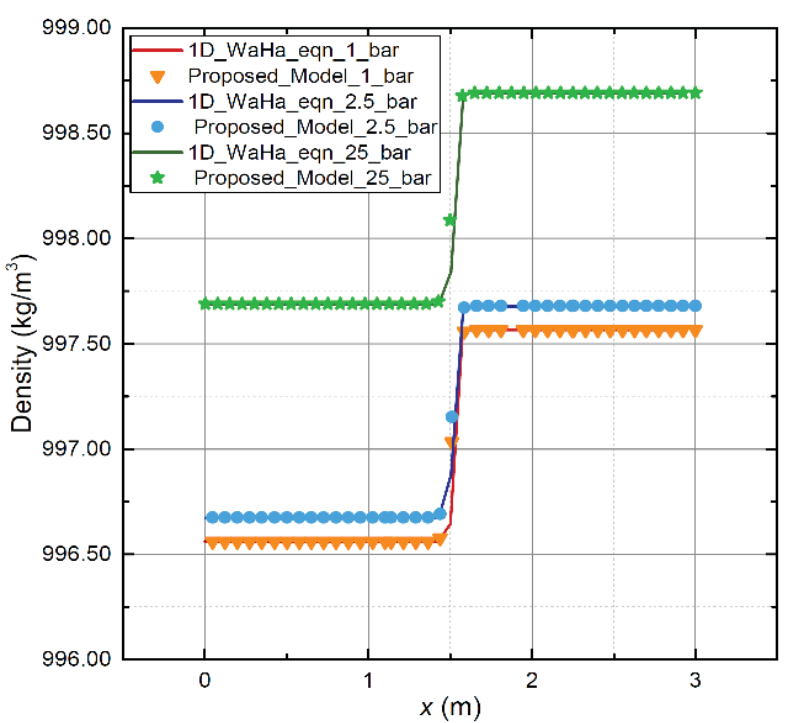

(b)

Fig. 4: Comparison of the Property Profiles at Different Operating Pressures from the Proposed Compressibility Model and the 1D Water Hammer Model

The surge in fluid pressure arising out of the sudden flow deceleration from the instantaneous valve closure is illustrated in Fig. 4(a). Due to the instantaneous closure of the valve, a compression pressure wave is generated at the valve (right boundary), which then propagates to the left at the sound speed. This compression front is visible in the form of discontinuity in the pressure profiles. The variation in the fluid density corresponding to the surge in pressure is presented in Fig. 4(b). From this plot, it can be seen that the compressibility of the liquid increases with an increase in the operating pressure range. The density predicted by the Tait EOS incorporated into the pressure-based incompressible algorithm is well-matched with the estimates using the compressible water-hammer formulation. An important observation regarding the profile resolution is that the discontinuity representing the compression front captured with the proposed model exhibits slight dissipation compared to the results of water hammer formulation. This can be attributed to the fact that the pressure-based solver introduces some amount of diffusion associated with the solver. The pressure-based solver does not enforce the acoustic time scale; instead, its time scale is based on the flow velocity, due to which the computational cost is drastically reduced. Whereas, this leads to a situation where the flow features related to acoustic wave propagation like the compression front, is not as sharp as those observed with a density-based solver which strictly enforces the acoustic time scale.

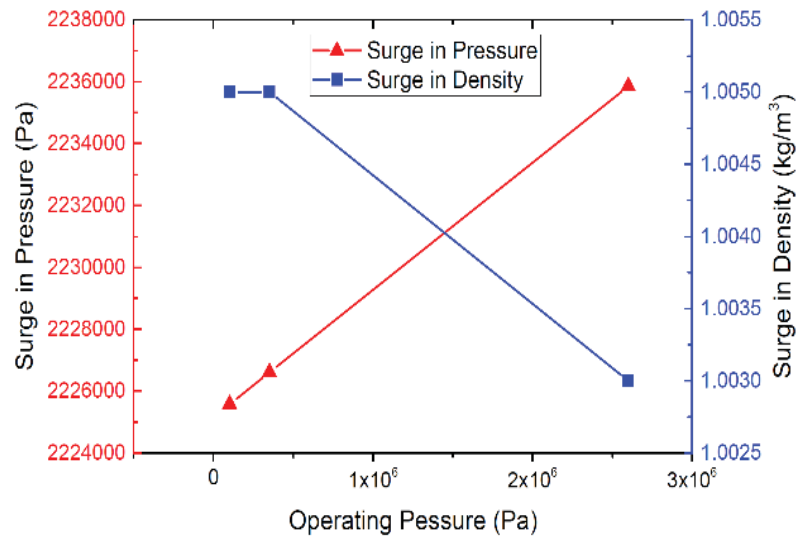

(a)

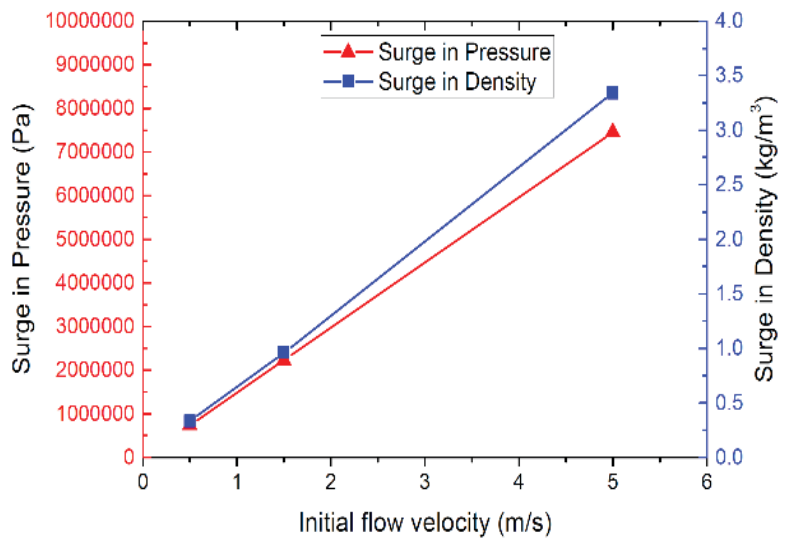

(b)

Fig. 5: Surge in Pressure and Density for Different Operating Pressures and Initial Flow Velocities 
The magnitude of the surge in the pressure and the associated density change due to the instantaneous valve closure, for an initial flow velocity of $1.5 \mathrm{~m} / \mathrm{s}$ at different operating pressures are shown in Fig. 5(a). The magnitude of the surge in the pressure is observed to increase with the rise in operating pressure, which is attributed to the high fluid density and speed of sound at higher pressure ranges. The change in density is, however, observed to drop with the rise in operating pressure. This is due to decrease in the ratio of the maximum surge pressure to the operating pressure with the increase in operating pressure. The estimated values of this ratio are $11.45,7.23$, and 1.82 , respectively, for the operating pressures of $1 \mathrm{bar}, 2.5 \mathrm{bar}$, and $25 \mathrm{bar}$. The importance of these ratios cannot be overemphasized as it is directly related to the safety of the pipe structure. For lower operating pressure, the pressure surge magnitudes are many times greater than the operating range of pressure, and if this information is not taken into account while deciding the design's safety factor, it could lead to catastrophic failure of the pipe structure.

The surge in pressure and the associated change in density with respect to the initial velocity of the flow in the pipe is displayed in Fig. 5(b). As expected, the magnitude of the surges in pressure and the density change is found to be proportional to the initial flow velocity. This is because fluid compression in the vicinity of the closed valve is higher with the greater deceleration of the fluid.

The instantaneous valve closure in an irrigation pipe is successfully simulated using the proposed pressure-based compressible-liquid flow model. Compressible modeling of the pipe fluid, i.e., liquid water, is accomplished by the solver using the Tait EOS. The accuracy and reliability of the proposed model are clearly revealed in comparison with the results of the classical water-hammer formulation. Compared to the computationally expensive density-based solvers, the proposed pressure-based model can be an alternate choice for the numerical modeling of low-speed compressible liquid flows, which utilizes the advantage of an incompressible flow solver. Provided that the appropriate EOS is available, the model can be extended to solve a large variety of compressible liquid flow problems.

\section{CONCLUSIONS}

A pressure-based compressible-liquid flow model is proposed for the simulation of isothermal lowspeed compressible-liquid flow problems. The model incorporates a liquid equation of state into a pressure- based incompressible solver algorithm. Instantaneous valve closure in an irrigation pipe is the test problem chosen and is numerically simulated using the proposed model. The model employs the Tait EOS for simulating compressibility effects in water, and a segregated pressure-based algorithm is used to simulate the problem on a 2D axisymmetric domain. The results are compared against those generated with the density-based one-dimensional water hammer formulation, which is used as the benchmark. The comparative evaluation reveals the capability of the proposed flow model to handle liquid compressibility with high accuracy. The surge in the pressure and variations in the density of the fluid is evaluated accurately. Variations in the magnitude of the surges in the pressure and the density changes over different operating pressure ranges revealed the importance of the ratio of maximum pressure to operating pressure in determining the safety of the structure. The model combines the advantages of an incompressible pressure-based solver and that of a density-based compressible solver. Thus, we conclude that the proposed pressure-based compressible-liquid flow model is a very accurate and inexpensive numerical tool for modeling isothermal low-speed compressible liquid flows. The model can be easily extended to simulate diverse compressible flow problems for various liquids subject to the availability of the relevant equation of state.

\section{ACKNOWLEDGMENT}

The authors are thankful to the Department of Aerospace Engineering, Indian Institute of Space Science and Technology for extending the support and encouragement in completing this study.

\section{REFERENCES}

Acharya S, Baliga BR, Karki K, Murthy JY, Prakash C, Vanka SP (2007) Pressure-based finite-volume methods in computational fluid dynamics, Journal of Heat Transfer 129(4): 407-424.

Bazargan-Lari MR, Kerachian R, Afshar H, Bashi-Azghadi SN (2013) Developing an optimal valve closing rule curve for real-time pressure control in pipes, Journal of Mechanical Science and Technology 27(1): 215-225.

Chandran J, Salih A (2019) A modified equation of state for water for a wide range of pressure and the concept of water shock tube, Fluid Phase Equilibria 483: 182-188.

Chen YS (1989) Compressible and incompressible flow computations with a pressure based method, 27th Aerospace Sciences Meeting: 286.

Chen ZJ, Przekwas AJ (2010) A coupled pressure-based computational method for incompressible/compressible flows, Journal of Computational Physics 229(24): 91509165. 
Choi DY, Kim J, Lee DJ, Kim D (2015) Pressure measurements with valve-induced transient flow in water pipelines, Urban Water Journal 12(3): 200-206.

Denner F (2018) Fully-coupled pressure-based algorithm for compressible flows: Linearisation and iterative solution strategies, Computers \& Fluids 175: 53-65.

Dymond JH, Malhotra R (1988) The Tait equation: 100 years on, International Journal of Thermophysics 9: 941-951.

Ghidaoui MS, Zhao M, McInnis D, Axworthy DH (2005) A review of water hammer theory and practice, Applied Mechanics Reviews, American Society of Mechanical Engineers 58: 49-76.

Guinot V (2003) Godunov-type schemes: An introduction for engineers, 1st edn. Elsevier.

Joukowsky D (1898) Contribution to the question of the conditions of multiplication and onset of conjugation in ciliates, $\mathrm{PhD}$ Thesis.

Kambrath PT, Cary AW (2014) Development of a coupled compressible pressure-based solver, 52nd Aerospace Sciences Meeting: 226.

Kodura A (2016) An analysis of the impact of valve closure time on the course of water hammer, Archives of Hydro-Engineering and Environmental Mechanics 63(1): 35-45.

Kraposhin MV, Banholzer M, Pfitzner M, Marchevsky IK (2018) A hybrid pressure-based solver for nonideal single-phase fluid flows at all speeds, International Journal for Numerical Methods in Fluids 88(2): 79-99.

Lourier JM, Di Domenico M, Noll B, Aigner M (2012) Implementation of an efficient pressure-based CFD solver for accurate thermoacoustic computations, 18th AIAA/ CEAS Aeroacoustics Conference (33rd AIAA Aeroacoustics Conference): 2089.

Miettinen A, Siikonen T (2015) Application of pressure-and densitybased methods for different flow speeds, International Journal for Numerical Methods in Fluids 79(5): 243-267.
Miller ST, Jasak H, Boger DA, Paterson EG, Nedungadi A (2013) A pressure-based, compressible, two-phase flow finite volume method for underwater explosions, Computers \& Fluids 87: 132-143.

Mitra AK, Rouleau WT (2005) Radial and axial variations in transient pressure waves transmitted through liquid transmission lines, Journal of Fluids Engineering 107: 105-111.

Moukalled F, Darwish M (2001) A high-resolution pressurebased algorithm for fluid flow at all speeds, Journal of Computational Physics 168(1): 101-130.

Report on "Pipe Distribution System for Irrigation" (1998) Indian National Committee on Irrigation and Drainage, New Delhi.

Rincon J, Elder R (1997) A high-resolution pressure-based method for compressible flows, Computers \& Fluids 26(3): 217-231.

Saurel R, Cocchi P, Butler PB (1999) Numerical study of cavitation in the wake of a hypervelocity underwater projectile, Journal of Propulsion and Power 15(4): 513-522.

Shterev KS, Stefanov SK (2010) Pressure based finite volume method for calculation of compressible viscous gas flows, Journal of Computational Physics 229(2): 461-480.

Students L (1903) General theory of disturbed water motion in pressure pipes (Water hammer) , LB students, Cooperative Union.

Tait PG (1888) Physics and Chemistry of the Voyage of H.M.S. Challenger, H.M.S.O., London, 2: 4.

Tijsseling AS, Bergant A (2007) Meshless computation of water hammer, 1st edn. Department of mathematics and computer science, University of technology.

Vardy AE, Hwang KL (2005) A characteristics model of transient friction in pipes, Journal of Hydraulic Research 29: 669-684.

Wylie EB, Streeter VL, Suo L (1993) Fluid transients in systems, 1st edn. Prentice Hall Englewood Cliffs, NJ.

Xiao CN, Denner F, van Wachem, Berend GM (2017) Fully-coupled pressure-based finite-volume framework for the simulation of fluid flows at all speeds in complex geometries, Journal of Computational Physics 346: 91-130. 